Original Research Article

\title{
Adverse events of albendazole due to mass drug administration
}

\author{
Pooja Agrawal*, Bhavana Srivastava, Reena Bhardwaj, Sanjay Gaur
}

Department of Pharmacology, Dr Susheela Tiwari Government Medical College and hospital, Haldwani, Uttarakhand, India

Received: 19 April 2017

Accepted: 16 May 2017

*Correspondence to:

Dr. Pooja Agrawal,

Email: vijenderagrawal@ gmail.com

Copyright: (C) the author(s), publisher and licensee Medip Academy. This is an openaccess article distributed under the terms of the Creative Commons Attribution NonCommercial License, which permits unrestricted noncommercial use, distribution, and reproduction in any medium, provided the original work is properly cited.

\begin{abstract}
Background: Soil-transmitted helminths are mostly prevalent in developing countries due to poor sanitation and lack of adequate clean water. The present study examines adverse events (AEs) experienced following administration of albendazole to children (2-19 Years) at Uttarakhand on national de-worming day.

Methods: Children were given single doses of albendazole on national deworming day. Some of children experienced adverse events and were admitted in hospital of Govt Medical college Haldwani (Uttarakhand). Data were collected and analyzed.

Results: Total twenty five children were admitted due to albendazole adverse events. Out of these $92 \%$ were female. Mean age of admitted children was 14.14 years with standard deviation 3.45. Mean onset of adverse events was 5.6 hours with standard deviation of 1.5 hours. All children were treated symptomatically and were discharged once they recovered. No fatality due to adverse events was observed. Average duration of stay in hospital was 3.4 days. Out of twenty five children $12 \%$ children reported four or more adverse events, $40 \%$ children reported three adverse events and $48 \%$ reported two adverse events. Out AEs, 33\% AEs were mild, 19\% AEs were moderate, 31\% AEs were severe and $17 \%$ AEs were serious. Abdominal pain was reported by $76 \%$, headache by $44 \%$, loss of consciousness by $32 \%$, vomiting by $28 \%$, nausea by $16 \%$, convulsions by $12 \%$, rashes by $8 \%$, fever by $8 \%$, and breathlessness by $14 \%$ and vertigo by $4 \%$.

Conclusions: The adverse events were mild to serious but transient, but all of them recovered after hospitalization. Therefore, it is imperative that mass drug administration programmes put in place surveillance measures in order to ensure timely detection, management and reporting of potential life threatening AEs.
\end{abstract}

Keywords: Albendazole, Adverse events, De-worming programme, Mass drug administration

\section{INTRODUCTION}

Hundreds of millions of people are infected with the common soil-transmitted helminths (STHs), namely hookworms (Ancylostoma duodenale and Necatoramericanus), Ascaris lumbricoides and Trichuris trichiura, many by multiple species concurrently. ${ }^{1-3}$ Taenia spp. infections are also widespread..$^{4,5}$ STHs and taeniasis/cysticercosis belong to the neglected tropical diseases and are responsible for mainly chronic and often inconspicuous morbidity. ${ }^{6,7}$ Iron-deficiency anemia, malnutrition, and impaired physical and cognitive development have all been attributed to STH infections. ${ }^{3,8}$ Taenia solium cysticercosis is a major cause of epilepsy and other neurological disorders in developing countries. ${ }^{9}$
The current strategy for STH control in highly endemic areas focuses on morbidity control through large-scale administration of single-dose anthelminthics to at-risk populations, particularly children. ${ }^{10,11}$ Due to the zoonotic nature of taeniasis/cysticercosis, its control must also include the veterinary sector. ${ }^{4,12,13}$ At present, only four drugs are recommended by the World Health Organization for treating STH infections. ${ }^{10,14}$ The global STH control relies on two of them-albendazole and mebendazole-both benzimidazole carbamates. Albendazole and mebendazole display a broad spectrum of activity and are administered orally, usually at a single dose of $400 \mathrm{mg}$ and $500 \mathrm{mg}$, respectively. ${ }^{10,14-17}$ Children below the age of 1 year and pregnant women in the first trimester of pregnancy are not eligible for treatment. ${ }^{10}$ 
Albendazole and mebendazole have been extensively used worldwide for more than 30 years, both as standalone treatments and, more recently, in combination with other drugs, e.g., praziquantel (against schistosomiasis and food-borne trematodiasis) or ivermectin (against lymphatic filariasis). ${ }^{7}$ Justification for the indiscriminate use of either drug is derived from high egg reduction rates achieved with both albendazole and mebendazole, and the assumption that morbidity is a function of infection intensity. ${ }^{18}$ However, a recent meta-analysis of randomized placebo-controlled single-dose drug efficacy trials pointed to a marked superiority of albendazole over mebendazole against hookworm, high efficacy in terms of cure rate of both drugs against $A$. lumbricoides, and disappointing efficacy of either drug against $T$. trichiura. $^{14}$

The new de-worming initiative of the Indian Health Ministry aims to de-worm all pre-school and school-age children between the ages of 1-19 years. Staring from the National De-worming Day on 10th February 2015, albendazole tablets are being given to all targeted children; half tablet to 1-2 years children and one full tablet for 2-19 years. The children who are left out are being covered by a mop-up round to be carried out till next four days. In the year 2016, also children were given albendazole on national De-worming Day (10 Feb 2016) in state of Uttrakhand. Some of children experienced serious adverse events like Convulsions/ loss of consciousness / breathlessness due to albendazole and were admitted in hospital of Govt Medical College Haldwani. Due to above some of children and parents were apprehensive, and children having mild AEs like nausea /headache/ rashes were also admitted in hospital. This hospital based study was carried out to study adverse events of albendazole in children due to mass drug administration for de-worming programme.

\section{METHODS}

This cross-sectional hospital based study was carried out in admitted children at hospital of Govt Medical College Haldwani due to adverse events (AEs) due to albendazole administration for national de-worming programme. This study was carried out from 11 February 2016 to 25 February 2016. Patients were referred from School/ Primary Health Center/Community Health Center. Oral informed consent was obtained from the study participants or their parents. A structured questionnaire was designed to study the adverse events of albendazole in admitted children. Questionnaire included (name, age, sex, onset of adverse events, clinical symptoms, outcome). Questionnaire was administered by investigator to all the admitted children or their parents in language they understand. Approval was taken from ethical committee of Govt Medical College Haldwani to carry out the study in admitted children. AEs were classified as defined in the WHO guidelines whereby mild AEs were defined as undesirable experiences associated with use of the antihelmithics but not affecting daily activities (e.g. playing) whereas moderate AEs were defined as those affecting performance of daily activities. Severe AEs were defined as those requiring total rest and/or medication while serious AEs were defined as those that were life-threatening requiring admission to hospital. ${ }^{19}$ Data were collected and analyzed in Microsoft Excel.

\section{RESULTS}

Total twenty five children were admitted due to albendazole adverse events. Out of these $2(8 \%)$ were male and 23 (92\%) were female. Mean age of admitted children was 14.14 years with standard deviation 3.45 and range was 3.5 years to 18 years. Mean onset of symptoms was 5.6 hours with standard deviation of 1.5 hours. All children were treated symptomatically and were discharged once they recovered. No fatality due to adverse events was observed. Average duration of stay in hospital was 3.4 days. Out of twenty five children 3 (12\%) children reported four or more adverse events, 10 (40\%) children reported three adverse events and 12 (48\%) reported two adverse events. Total sixty four adverse events were reported. Out AEs, 33\% AEs were mild ,19\% AEs were moderate, $31 \%$ AEs were severe and $17 \%$ AEs were serious (Figure 1).

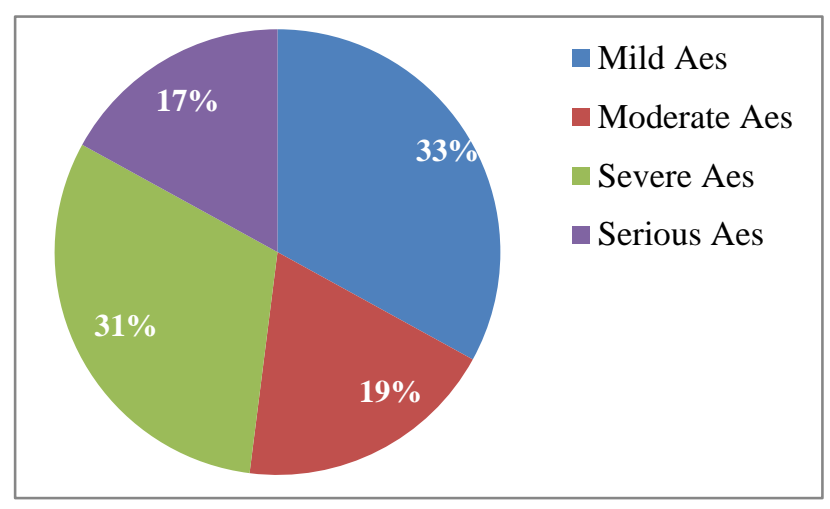

Figure 1: Nature of adverse events (Aes).

Table 1: Adverse events due to Albendazole Mass drug administration.

\begin{tabular}{|ll|l|}
\hline Type of adverse event & Frequency & Percentage \\
\hline Abdominal pain & 19 & $76 \%$, \\
\hline Headache & 11 & $44 \%$ \\
\hline Loss of consciousness & 8 & $32 \%$ \\
\hline Vomiting & 7 & $28 \%$ \\
\hline Nausea & 4 & $16 \%$ \\
\hline Convulsions & 3 & $12 \%$ \\
\hline Rashes & 2 & $8 \%$ \\
\hline Fever & 2 & $8 \%$ \\
\hline Breathlessness & 1 & $4 \%$ \\
\hline Vertigo & 1 & $4 \%$ \\
\hline
\end{tabular}

Abdominal pain was reported by $19(76 \%)$, headache by $11(44 \%)$, loss of consciousness by $8(32 \%)$, vomiting by 
$7(28 \%)$, nausea by $4(16 \%)$, convulsions by $3(12 \%)$, rashes by $2(8 \%)$, fever by $2(8 \%)$, breathlessness by 1 (4\%) and vertigo by $1(4 \%)$ (Table 1$)$.

\section{DISCUSSION}

The spectrums of the adverse events of drugs are not always same between different ethnic groups, and it can be influenced by the genetic factors and even the temporary conditions of children at the de-worming activity. The result of this study shows the frequencies of the adverse effects of the albendazole used in national deworming programme among children in an area Uttrakhand. In mass de-worming programme in Central Kenya with albendazole cough was reported more frequently. Cough is a general reaction and can be associated with any drug especially in young children. ${ }^{20}$ In our study cough was not reported at all as AEs. Albendazole provides safe and highly effective therapy against worm infestation. ${ }^{21}$ It has only limited solubility in water. After a $400 \mathrm{mg}$ oral dose, albendazole cannot be detected in plasma, due to low absorbance of the drug from the intestines. ${ }^{22}$ The metabolites are excreted mainly in the urine. Albendazole is said to produce few adverse events when used for short-term therapy of worm infestations. Transient abdominal pain, diarrhea, nausea, dizziness, and headache occur occasionally. ${ }^{23}$ Indeed, a review by Horton, reported that incidences of side effects associated with albendazole at the doses used for the treatment of intestinal helminths are very low, mild and self-limiting with only gastrointestinal side effects occurring with an overall frequency of just greater than $1 \%{ }^{24}$ Similar adverse events were also reported in our study. However in our study some of children reported serious adverse events like loss of consciousness, convulsions but all of them recovered after hospitalization and in none of children convulsion and loss of consciousness was observed during hospital stay. The reasons for the variations in proportions of adverse events experienced after antihelminthic treatment could probably be due to dissimilar intensities of parasites as well as differences in socio-economic status, types of foods and environmental conditions associated with the diverse populations. Mass use of praziquantel in areas where cysticercosis is endemic can trigger seizures in persons with latent brain cysts a potential concern for mass deworming campaigns involving albendazole. A case of unmasking of neurocysticercosis was reported in Peru due to albendazole administration. ${ }^{23,25}$ Those designing or executing deworming programs should carefully consider the possibility of this complication. Therefore, it is imperative that mass drug administration programmes put in place surveillance measures in order to ensure timely detection, management and reporting of potential life threatening AEs.

\section{CONCLUSIONS}

This study shows that albendazole have mild as well as serious adverse events. Trained school teachers can however use the albendazole in MDA for control of STHs since schools provide an efficient and effective channel to reach large portions of the population within a short time, which makes a programme cost effective. However, the health/medical personnel should be involved in the programme so that they can give moral support and be on standby to handle any serious adverse events should they arise. This approach is cost effective and should be adopted for a national deworming programme. Therefore, it is imperative that mass drug administration programmes put in place surveillance measures in order to ensure timely detection, management and reporting of potential life threatening AEs.

Funding: No funding sources

Conflict of interest: None declared

Ethical approval: The study was approved by the Institutional Ethics Committee

\section{REFERENCES}

1. Steinmann $\mathrm{P}$, Du ZW, Wang LB, Wang XZ, Jiang JY. Extensive multiparasitism in a village of Yunnan province, People's Republic of China, revealed by a suite of diagnostic methods. Am J Trop Med Hyg. 2008;78:760-9.

2. Steinmann $\mathrm{P}$, Utzinger $\mathrm{J}, \mathrm{Du} \mathrm{ZW}$, Zhou XN. Multiparasitism: a neglected reality on global, regional and local scale. Adv Parasitol. 2010;73:2150.

3. Pullan R, Brooker S. The health impact of polyparasitism in humans: are we under-estimating the burden of parasitic diseases? Parasitology. 2008;135:783-94.

4. Willingham AL, Engels D. Control of Taenia solium cysticercosis/taeniosis. Adv Parasitol. 2006;61:50966.

5. Craig P, Ito A. Intestinal cestodes. Curr Opin Infect Dis. 2007;20:524-32.

6. Ezeamama AE, Friedman JF, Olveda RM, Acosta LP, Kurtis JD, et al. Functional significance of lowintensity polyparasite helminth infections in anemia. J Infect Dis. 2005;192:2160-70.

7. Hotez PJ, Molyneux DH, Fenwick A, Kumaresan J, Ehrlich Sachs S, et al. Control of neglected tropical diseases. N Engl J Med. 2007;357:1018-27.

8. Brooker S, Hotez PJ, Bundy DAP. Hookwormrelated anaemia among pregnant women: a systematic review. PLoS Negl Trop Dis. 2008;2:e291.

9. Garcia HH, Moro PL, Schantz PM. Zoonotic helminth infections of humans: echinococcosis, cysticercosis and fascioliasis. Curr Opin Infect Dis. 2007;20:489-94.

10. WHO. Preventive chemotherapy in human helminthiasis. Coordinated use of anthelminthic drugs in control interventions: a manual for health professionals and programme managers. Geneva: World Health Organization; 2006:1-62. 
11. Hotez PJ, Fenwick A, Savioli L, Molyneux DH. Rescuing the bottom billion through control of neglected tropical diseases. Lancet. 2009;373:1570-5.

12. Montresor A, Palmer K. Taeniasis/cysticercosis trend worldwide and rationale for control. Parasitol Int. 2006;55:S301-3.

13. OIE/WHO/FAO. WHO/FAO/OIE guidelines for the surveillance, prevention and control of taeniasis/cysticercosis; In: Murrell KD, editor. Paris: World Organization for Animal Health. 2005:1-139.

14. Keiser J, Utzinger J. Efficacy of current drugs against soil-transmitted helminth infections: systematic review and meta-analysis. JAMA. 2008;299:1937-48.

15. Horton J. Albendazole: a broad spectrum anthelminthic for treatment of individuals and populations. Curr Opin Infect Dis. 2002;15:599-608.

16. Chavarria AP, Swartzwelder JC, Villarejos VM, Zeledon R. Mebendazole, an effective broadspectrum anthelmintic. The American journal of tropical medicine and hygiene. 1973 Sep;22(5):5925.

17. Geary TG, Woo K, McCarthy JS, Mackenzie CD, Horton $\mathbf{J}$, et al. Unresolved issues in anthelmintic pharmacology for helminthiases of humans. Int $\mathrm{J}$ Parasitol. 2010;40:1-13.

18. Albonico M, Allen H, Chitsulo L, Engels D, Gabrielli AF, et al. Controlling soil-transmitted helminthiasis in pre-school-age children through preventive chemotherapy. PLoS Negl Trop Dis. 2008;2:e126.

19. World Health Organization. Assuring safety of preventive chemotherapy interventions for the control of neglected tropical diseases; 2011:1-54.
20. Njomo DW, Tomono $\mathrm{N}$, Ng'ethe $\mathrm{M}$, Mitsui $\mathrm{Y}$, Josyline KC, Mwandawiro CS. The adverse effects of albendazole and praziquantel in mass drug administration by trained schoolteachers. Afr $\mathbf{J}$ Health Sci. 2010;16:10-4.

21. Marriner SE, Morris DL, Dickson B, Bogan JA. Pharmacokinetics of albendazole in man. European Journal of Clinical Pharmacology. 1986;30:705-8.

22. Urbani C, Albendazoleonico M. Anthelminthic drug safety and drug administration in the control of soil transmitted helminthiasis in community campaigns. Acta Tropica. 2003;86:215-21.

23. Flisser A, Madrazo I, Plancarte A. Neurological symptoms in occult neurocysticercosis after single taeniacidal dose ofpraziquantel. Lancet. 1993;342:748.

24. Horton J. Albendazole: a review of anthelmintic efficacy and safety in humans. Parasitology. 2000;121:S113-32.

25. Garcia HH. Isidro Gonzalez Neurocysticercosis Uncovered by Single-Dose Albendazole (Letter to Editor). N Engl J Med. 2007;356;12:1277.

Cite this article as: Agrawal $\mathrm{P}$, Srivastava B, Bhardwaj R, Gaur S. Adverse events of albendazole due to mass drug administration. Int $\mathrm{J}$ Basic Clin Pharmacol 2017;6:1674-7. 